\title{
Entropy Production In Thermodynamic Climate Models
}

\author{
T. Pujol, J. E. Llebot \\ Grup de Física, Dept. de Ciències Ambientals, Universitat de Girona, Girona, Spain
}

Registration Number 767

\begin{abstract}
We present a non-equilibrium theory in a system with heat and radiative fluxes. The obtained expression for the entropy production is applied to a simple one-dimensional climate model based on the first law of thermodynamics. In the model, the dissipative fluxes are assumed to be independent variables, following the criteria of the Extended Irreversible Thermodynamics (EIT) that enlarges, in reference to the classical expression, the applicability of a macroscopic thermodynamic theory for systems far from equilibrium. We analyze the second differential of the classical and the generalized entropy as a criteria of stability of the steady states. Finally, the extreme state is obtained using variational techniques and observing that the system is close to the maximum dissipation rate.
\end{abstract}

\section{Introduction}

In the late 60 's and in the 70's the development of one-dimensional climate models based on the first law of thermodynamics had its apogee. The importance of these simple models was centered in the possibility to understand the weight of the parameters that intervene in the climate system [1-5]. These previous works led scientists to create more sophisticated climate models with two and three dimensional variables [6-10] until arriving to the present General Circulation Models (GCM), that suffer of a high level of complexity with the intention to simulate all the physical processes present in the atmosphere. Thus, different types of GCMs have been used to diagnose the laws that govern the interactions between the elements of the climate system and to predict possible climate evolution in different possible scenarios [11-12]. However, due to their complexity these models do not permit a direct application of new concepts in the study of climate. On the other hand, the one-dimensional climate models do not give quantitative solution for the climate but are very useful tools for introducing new parameters and for observing how the new terms affect and modify the state of the system. Following this way, some authors have recently studied the role of entropy in climate. $\mathrm{Li}$ and Chýlek [13] have used a one-dimensional latitudinaldependent climate model developed by North [3] for obtaining the expression of the 
rate of entropy production. Furthermore, $\mathrm{Li}$ et al. [14] consider a one-dimensional radiative-convective model to observe the vertical distribution of the rate of entropy production. Looking for the possibility of an extremal of the dissipation in the climate system, formerly claimed by Paltridge [15-18], Stephens and O'Brien [19] have calculated the contribution of the radiative flux of entropy using satellite data and comparing them with analytical results. Later, O'Brien and Stephens [20] use a Paltridge box model pointing out that it follows the principle of maximum dissipation proposed by Ziegler [21]. Paltridge developed a box-model assuming that the climate system is close to the maximum dissipation rate. With this constraint, the Paltridge model gives values of temperature and cloud cover extraordinarily close to the real ones as has been shown by Grassl [22], introducing the ice-albedo feedback.

Therefore, our purpose is to formulate a non-equilibrium climate model in order to obtain a general expression of the rate of entropy production outside equilibrium. We will follow Nicolis and Nicolis [23] to explore wether or not the climate system is governed by an extremal principle related with the entropy production. Moreover, with the aim of doing a rigorous deduction according to the most recent macroscopic theories, we will assume the dissipative fluxes of the system to be independent variables. Thus, a macroscopic expression will be obtained in the framework of the Extended Irreversible Thermodynamics (EIT). This theory has been successfully used in a wide range of macroscopic systems but it has never been used in climatic systems. In Section 2 , a general expression of the rate of the generalized entropy production outside equilibrium is obtained in a system with heat and radiative fluxes. In Section 3, a one-dimensional climate model is developed following the one proposed by North [3-4] with the intention of evaluating a qualitative behaviour of the rate of entropy production. In the horizontal diffusive model used the horizontal heat flux is approximated using a Fourier's law type equation. As this model is vertically averaged, it is not necessary to know the radiation flux, only its divergence. We will introduce, then, an expression for the radiative flux in order to apply the development proposed by EIT. When its divergence is averaged vertically the parameterization of North's model is obtained. Then we will be able to define a generalized entropy with an expected larger range of validity than the expressions used before. Both, the rate of the classical and extended entropy production, are compared in Section 4 where we also study their time evolution for different cases. They present different behaviours when different initial conditions are chosen. Thus, at the steady state a maximum or a minimum in the rate of entropy production is obtained in function of the values assumed for the initial conditions. Even, the attainment of this extremal at the steady state in reference to the time evolution is not always fulfilled.

In Section 5, we discuss the possibility of using the second differential of the generalized entropy as a stability criteria for the climate system. The classical expression has been largely used in thermodynamic fluid systems for obtaining the stability at stationary conditions. This hypothesis applied to the climate has been initially proposed by Nicolis and Nicolis [23]. The second differential of the generalized entropy has been studied by Jou et al. [24] where the convexity requirement implies a maximum permissible value for the dissipative fluxes, directly related with the range of applicability of the EIT theory. Here, the classical and the generalized expression of the second 
differential of the entropy are studied and their obviously different behaviour is emphasized.

On the other hand, a maximum entropy production at the steady state can be obtained which is a minimum in reference to the time evolution. It is due to the existence of an extremal entropy production for fixed conditions, which value has been calculated at the steady state using variational techniques (Sect. 6). We have analyzed three expressions related to the rate of entropy production: the global, the thermal and the difference between the radiative flux and the radiative energy contribution of entropy. Furthermore, the time evolution of the rate of entropy production is shown in comparison with the extremal value obtained using the variational principle. This calculation is only acceptable for a time evolution close to the steady state presuming that the extremal conditions are also applicable not very far from the equilibrium. However, a variational principle outside the steady state is applied with some additional restrictions, obtaining a good approach of the extremal values to the observed ones along the time evolution. In Section 7, finally, we conclude the work with the discussion of the results obtained.

\section{Generalized entropy production}

In the last years the common acceptance of studying the climate through complex 2-dimensional and 3-dimensional models, to take into account the great quantity of physical phenomena present in the atmospheric system, has not permitted to introduce new concepts. There have been some attempts to investigate the role of the rate of entropy production in the climate system using simple 1-dimensional models [13-14, 23] or using it as a fundamental part for the calculation of the variables in a box model $[15-20,22]$. In a closed thermodynamic system the study of the behaviour of the rate of entropy production has been extensively used [25]. However, in an open system with a radiation field (e.g., the climate) it is still unclear how the entropy production will evolve and wether it is of importance for the states reached by the system. Thus, it is not clear wether or not the system follows an extreme principle at the steady state [23, 26, 27]; also it is unclear wether the rate of entropy production can be expressed as a bilinear form, though there have been attempts to do so [28, 29]. Even in the treatment of the entropy on the climate, some authors have considered only the thermal part [23] pointing out that the radiative contribution has no relevance on the dynamics of the system and, in consequence, on the state that it is led to [20]. The assumption of a thermodynamic 1-dimensional model permits a great technical simplification, with only three thermodynamics variables: the temperature, the heat and the radiative fluxes. However, there have been attempts to find an extremal behaviour of the rate of entropy production in simple dynamic models $[30,31]$ with the intention to obtain a principle equivalent to the principle of minimum entropy production proposed by Prigogine [32], not necessarily restricted to closed systems [25]. Nevertheless, the existence of the radiation field in the climate implies a great difficulty and raises doubts in the success of this task.

Here, we will obtain a general expression of entropy production for non-equilibrium situations, assuming an open system with two perpendicular fluxes, the heat flux and 
the radiative flux. When the system is outside the steady state, the classical phenomenological laws are not satisfied and we consider the fluxes as independent variables. The thermodynamic theory that assumes the introduction of the fluxes as independent variables has been called Extended Irreversible Thermodynamics (EIT) because, in principle, it is an extension of the classical non-equilibrium thermodynamics based on the local equilibrium hypothesis. EIT mainly deals with a generalized entropy, obtained from a generalized Gibbs equation. This generalized thermodynamics has enlarged the applicability of a macroscopic theory in the study of multiple physical systems (e.g., fluids, rheology, cosmology, ...) and has obtained significant agreements with mesoscopic theories (kinetic theory, statistical mechanics and information theory) [24]. Now, we try to use it in a climatic system.

We assume the generalized expression of the rate of entropy production to be not only a function on the extensive variables as the classical expression but also of the dissipative fluxes. Then, $s^{*}=s_{r}^{*}+s_{m}^{*}$ where the subscripts $r$ and $m$ indicate the radiation and the matter part respectively and ${ }^{*}$ denotes the generalized entropy. With the assumption that $s^{*}=s^{*}(u, \boldsymbol{Q}, \boldsymbol{R})$, the generalized Gibbs equation has the form

$$
\rho d s^{*}=+\frac{\rho}{T} d u-\frac{1}{T} A_{Q} \cdot d Q-\frac{1}{T} A_{R} \cdot d R,
$$

where $Q$ is the heat flux and $R$ the radiative flux, $u$ is the internal energy of the system and $\rho$ its density. Many authors consider only as acceptable a Gibbs equation related to the respective material parts of the internal energy and entropy but, as Fort and Llebot [33] remark, it is possible to assume a Gibbs equation to hold for the total, i.e. radiation including entropy and internal energy. However, if we only adopt a Gibbs equation for the material part, the expression of the rate of entropy production that we will obtain, would be modified by the introduction of local time derivatives in the material part of the internal energy and entropy. However, once calculated their values, in the cases assumed in Section 4, and being applied to the climate system, we would observe a considerable lower contribution than the additional terms obtained using the EIT theory.

The first term on the rhs of (1) corresponds to the classical entropy. The coefficients $A_{Q}$ and $A_{R}$ are obtained relating (1) to the balance equation for the entropy, as follows:

$$
\rho \frac{d s^{*}}{d t}=-\nabla \cdot J_{s}+\sigma
$$

Here $J_{s}$ is the entropy flux and $\sigma$ the rate of entropy production. From (1) and (2), $\sigma$ can be calculated as

$$
\sigma=\nabla \cdot J_{s}-\frac{\rho}{T} \frac{d u}{d t}-\frac{A_{Q}}{T} \cdot \frac{d Q}{d t}-\frac{A_{R}}{T} \cdot \frac{d R}{d t}
$$

The variation of the internal energy of the system is related to the dissipative fluxes 
through the first law of thermodynamics as follows

$$
\rho \frac{d u}{d t}=-\nabla \cdot Q-\nabla \cdot R
$$

We divide the entropy flux into a thermal and radiative part

$$
J_{s}=\frac{Q}{T}+H
$$

where $Q / T$ and $\boldsymbol{H}$ are the thermal and the radiative part respectively. The main problem of a thermodynamic treatment of the radiation is that the radiative flux of entropy is not linearly related with the radiative flux of energy [34]. However, in next sections we will assume a proportionality of both expressions through effective temperatures chosen conveniently $[13,27]$. With (5) and (4), equation (3) can be expressed as

$$
\sigma=Q \cdot \nabla \frac{1}{T}-\frac{A_{Q}}{T} \cdot \frac{d Q}{d t}+\nabla \cdot H-\frac{\nabla \cdot R}{T}-\frac{A_{R}}{T} \cdot \frac{d R}{d t} .
$$

For the coefficients $\boldsymbol{A}_{\boldsymbol{Q}}$ and $\boldsymbol{A}_{\boldsymbol{R}}$, we assume a linear dependence on the fluxes [24]:

$$
\begin{aligned}
& \boldsymbol{A}_{\boldsymbol{Q}}=\alpha_{\boldsymbol{Q}} \boldsymbol{Q}+\alpha_{Q R} \boldsymbol{R}, \\
& \boldsymbol{A}_{\boldsymbol{R}}=\alpha_{\boldsymbol{R}} \boldsymbol{R}+\alpha_{\boldsymbol{R} Q} \boldsymbol{Q} .
\end{aligned}
$$

The coefficients $\alpha_{Q R}$ and $\alpha_{R Q}$ are identically zero because the two fluxes $\boldsymbol{Q}$ and $\boldsymbol{R}$ in the simple 1-dimensional model are assumed to be perpendiculars ( $\boldsymbol{Q}$ horizontal heat flux, $\boldsymbol{R}$ vertical radiation flux) [13]. Therefore, equation (6) takes the form

$$
\sigma=Q \cdot\left(\nabla \frac{1}{T}-\frac{\alpha_{Q}}{T} \frac{d Q}{d t}\right)+R \cdot\left(\nabla \frac{1}{T}-\frac{\alpha_{R}}{T} \frac{d R}{d t}\right)+\nabla \cdot H-\nabla \cdot\left(\frac{R}{T}\right) .
$$

Note that we here implicitly have assumed an independence between the fluxes and the temperature. Let us now consider an evolution equation commonly used for the heat flux and including a relaxation time $\tau_{Q}$,

$$
\tau_{Q} \frac{\partial Q}{\partial t}+Q=-\lambda^{\prime} \nabla^{\nabla T}
$$

where $\lambda^{\prime}{ }_{Q}$ is the thermal conductivity of the system.

Equation (9) is a Maxwell-Cattaneo equation [24, 35] that in the steady state has the form of Fourier's law. For the radiation field, we accept a similar form to (9) [33, 36-37],

$$
\tau_{R} \frac{\partial \boldsymbol{R}}{\partial t}+\boldsymbol{R}=-\lambda_{R} \nabla T
$$

In fact (10) can be directly deduced from the radiative transfer equation choosing suitably the values of $\lambda_{R}$ and $\tau_{R}$. An expression similar to (10) has been used by Fort and 
Llebot [33] applying a generalized expression for the entropy in studying the radiative transfer equation.

With (9) and (10) and with the intention of obtaining a bilinear form of $\sigma$ in terms of the fluxes, the coefficients $\alpha_{Q}$ and $\alpha_{R}$ are identified as

$$
\begin{aligned}
& \alpha_{Q}=\frac{\tau_{Q}}{\lambda_{Q}^{\prime} T}, \\
& \alpha_{R}=\frac{\tau_{R}}{\lambda_{R} T} .
\end{aligned}
$$

The generalized expression of entropy production is then

$$
\sigma=\frac{\boldsymbol{Q} \cdot \boldsymbol{Q}}{\lambda^{\prime}{ }_{Q} T^{2}}+\frac{\boldsymbol{R} \cdot \boldsymbol{R}}{\lambda_{\boldsymbol{R}} T^{2}}+\nabla \cdot \boldsymbol{H}-\nabla \cdot\left(\frac{\boldsymbol{R}}{T}\right)
$$

However, this is not a bilinear form for $\sigma$ due to the non-linear dependence of $\boldsymbol{H}$ in $\boldsymbol{R}$ [34]. Nevertheless, some authors have accepted a bilinear form at the steady state, developing the radiative terms on the wavelength, the solid angle and the spectral energy radiance $[28,29]$.

\section{Entropy production in the climate model}

In Section 2 we have obtained the rate of a generalized entropy production outside equilibrium in a system where the dissipative fluxes, the thermal flux and the radiative flux, are perpendicular to each other. In order to consider how this expression modifies the results found by the classical equation [13], it would be of interest to apply it to a simple climate model. The one-dimensional latitudinal-dependence Energy Balance Models (EBM) are the adequate tools for using (12) because these models do not take into account the dynamics of the system. Here, we will use a diffusive EBM model developed by North [4]. This branch of EBM models assumes the heat flux to be described by Fourier's law with a definite value of the conductivity-diffusivity of the system $[13,23]$. The simplicity of these models encouraged many authors to use them for observing the behaviour of variables such as the entropy in the climate system. Following Budyko [1] we consider a linear parameterization of the long-wave radiative divergence in function of the temperature as

$$
\nabla \cdot \boldsymbol{R}_{L}=A+B T
$$

$A$ and $B$ being empirical constants and $T$ the surface temperature in degrees Celsius. Equation (13) can be accepted in the atmospheric range of temperatures, taking into account the dependence of the atmospheric emissivity on them. For the short-wave radiative divergence we have

$$
\nabla \cdot R_{s}=S a,
$$

where $S$ represents the insulation function, the solar constant for the whole system, and $a$ the coalbedo $(a=1-\alpha$, with $\alpha$ the albedo). 
Despite the fact that the diffusive EBMs seem to be the adequate models to develop the formulation done in Section 2, these models can not be used to obtain the rate of entropy production outside equilibrium. The reason is that these models do not consider an explicit formula for the radiative flux. Aiming to overcome this problem, we introduce a former 2-dimensional (vertical and latitudinal) model which after integration along the vertical axis will give us the classical diffusive EBM. Then, we postulate a dependence for the three thermodynamic variables on the coordinates $(t, x, r)$, with $t$ as the time, $x$ the sine of the latitude $(0 \leq x \leq 1)$ and $r$ the axial radius, as

$$
\begin{aligned}
& T(t, x, r)=T(t, x) c_{1} \frac{e^{-H / H_{p}}}{R_{t}}, \\
& Q(t, x, r)=Q(t, x) c_{1} \frac{e^{-H / H_{p}}}{r} \\
& \boldsymbol{R}(t, x, r)=\boldsymbol{R}(t, x) f(r) .
\end{aligned}
$$

Here the constant $c_{1}$ has the unit of length and the dimensionless function $f(r)$ denotes the behaviour of the attenuation of the radiation along the vertical. $H$ is the width of the atmosphere and $H_{p}$ is a constant with unit of length that will correspond to the height where the radiative divergence takes its maximum value. $R_{\mathrm{t}}$ is the surface radius of the earth. Although in (15a) a dependence on $r$ for the temperature has not been assumed, it does not invalidate equations $(15 \mathrm{a}-\mathrm{c})$. These have been chosen according to the condition to reach to the classical models after integrating along the vertical with a plausible value for the radiative flux.

We have also used the gradient operator in spherical coordinates, that takes the form

$$
\begin{aligned}
& \nabla=a_{x} \frac{\left(1-x^{2}\right)^{1 / 2}}{r} \frac{\partial}{\partial x}+a_{r} \frac{\partial}{\partial r} \\
& \nabla \cdot=a_{x} \frac{1}{r} \frac{\partial\left(1-x^{2}\right)^{1 / 2}}{\partial x}+a_{r} \frac{1}{r^{2}} \frac{\partial r^{2}}{\partial r}
\end{aligned}
$$

where $a_{x}$ and $a_{r}$ are unit vectors towards the pole and the zenith respectively.

Using (15b) and (16a), equation (9) has the following form

$$
\left\{\tau_{Q} \frac{\partial Q}{\partial t}+Q=-\lambda_{Q}\left(1-x^{2}\right)^{1 / 2} \frac{\partial T}{\partial x}\right\} c_{1} \frac{e^{-H / H_{p}}}{r}
$$

where we have redefined the conductivity of the system as

$$
\lambda_{Q}=\frac{\lambda_{Q}^{\prime}}{R_{t}}
$$

From (17) we obtain

$$
\tau_{\boldsymbol{Q}} \frac{\partial \boldsymbol{Q}}{\partial t}+\boldsymbol{Q}=-\dot{\lambda_{\boldsymbol{Q}}} \nabla T
$$


with $\nabla$ being now a dimensionless operator. A similar equation to (19) has been used instead of Fourier's law in a EBM giving self-sustained oscillations for a definite range of response time [38]. Now, we introduce the first law of thermodynamics, equation (4), used in the EBM models

$$
C \frac{\partial T}{\partial t}=-\nabla \cdot Q-(A+B T-S a)
$$

Here $C$ is the heat capacity of the system and

$$
\boldsymbol{R}(t, x)=\boldsymbol{R}_{L}(t, x)+\boldsymbol{R}_{S}(t, x)=(A+B T-S a) a_{r},
$$

with $f(r)$ being due to the condition

$$
f\left(R_{f}\right) R_{f}^{2}=c_{1} e^{-H / H_{p}} H,
$$

where $R_{f}$ is the terrestrial radius at the top of the atmosphere, assuming for convenience $f\left(r=R_{t}\right)=0$. Going back to (10) and following Ciancio and Verhás [36-37], we obtain for a very anisotropic radiation field

$$
\begin{aligned}
\tau_{R} & =\frac{1}{\sigma_{A} c}, \\
\lambda_{R} & =\frac{c}{\sigma_{A}} \frac{d U_{R}}{d T},
\end{aligned}
$$

with $c$ being the velocity of light, $\sigma_{A}$ the absorption coefficient and $U_{R}$ the internal radiative energy per unit volume of the atmosphere, that can be expressed as

$$
U_{R}=\frac{1}{c} \int_{\lambda} d \lambda \int_{\Omega} d \Omega I(\lambda) .
$$

Here $\lambda$ is the wave-length, $\Omega$ the solid angle and $I(\lambda)$ the spectral energy radiance. The results for the long-wave and the short-wave radiation obtained from (25) taking into account the different values of the solid angle are

$$
\begin{gathered}
U_{L R}=\frac{2 \boldsymbol{R}_{L} \cdot \boldsymbol{a}_{r}}{c}, \\
U_{S R}=\frac{\boldsymbol{R}_{S} \cdot \boldsymbol{a}_{r}}{c} .
\end{gathered}
$$

As above, the subscripts $L$ and $S$ correspond to the long-wave and the short-wave radiation respectively.

Due to (26a-b), (21) and (15a, c) $\lambda_{R}$ takes the form

$$
\lambda_{R}=\frac{1}{\sigma_{A}}\left(2 B-S \frac{d a}{d T}\right) \frac{f(r) R_{t}}{c_{1} e^{-H / H_{p}}}
$$

where $T$ is only a function of $t$ and $x$.

J. Non-Equilib. Thermodyn. $\cdot 1998 \cdot$ Vol. $23 \cdot$ No. 1 
The short-wave term can be calculated from the expression assumed for the coalbedo $a$ in the diffusive EBM models

$$
a\left(x_{s}, x\right)=a(x) \theta\left(x_{s}-x\right)+b_{o} \theta\left(x-x_{s}\right),
$$

where $\theta$ is the step function of Heaviside and $x_{s}$ the sine of the latitude of the ice-sheet edge. Above this point the coalbedo is constant and equal to the ice value, $b_{0}$. Then we have

$$
\frac{d a}{d T}=\left(a(x)-b_{o}\right) \delta\left(x_{s}-x\right) \frac{d x_{s}}{d \grave{T}^{\prime}}
$$

with $\delta\left(x_{s}-x\right)$ being the Dirac delta function, centered in $x_{s}$ that is directly related with the temperature and postulated to be the same as the latitude where

$$
T\left(x_{s}\right)=-10^{\circ} \mathrm{C} \text {. }
$$

The latitudinal dependence of the EBM assumed in the present paper follows the development done by North [3-4]. Thus, assuming symmetric hemispheres, the temperature can be expressed as an expansion on Legendre polynomials cutting off until second order

$$
T(t, x)=T_{0}(t)+P_{2}(x) T_{2}(x),
$$

where $P_{2}(x)$ is the second order Legendre polynomial, $T_{0}$ the global mean temperature and $T_{2}$ is 1.5 times the difference of pole-equator temperatures. For the latitudinal dependence of the coalbedo and for the insulation function, an expression in terms of Legendre polynomials is also taken into account

$$
\begin{aligned}
& a(x)=a_{0}+a_{2} P_{2}(x), \\
& S(x)=S_{c}\left(1+S_{2} P_{2}(x)\right) .
\end{aligned}
$$

Here $S_{c}$ is the solar constant. In order to evaluate the weight of the long-wave and the short-wave radiation field in $\lambda_{R}$, we take a meridional integration of the short-wave radiation contribution in (27). In a climatic state equivalent to the present $\left(T_{0}=14.9^{\circ} \mathrm{C}\right.$, $T_{2}=-28.2^{\circ} \mathrm{C}, x_{s}=0.96$ with $b_{0}=0.38, a_{0}=0.697, a_{2}=-0.0779, S_{2}=-0.477$, $S_{c}=340 \mathrm{~W} \mathrm{~m}^{-2}, \quad A=214.2 \mathrm{~W} \mathrm{~m}^{-2}, B=1.575 \mathrm{~W} \mathrm{~m}^{-2} \mathrm{~K}^{-1}, \lambda_{Q}=0.591 \mathrm{~W} \mathrm{~m}^{-2} \mathrm{~K}^{-1}$ and $C=3.13810^{+8} \mathrm{~J} \mathrm{~m}^{-2} \mathrm{~K}^{-1}$ ), from (29), taking into account the condition (30) for the beginning of the ice-sheet, we have

$$
\int_{0}^{1} d x S \frac{d a}{d T}=0.59 \mathrm{Wm}^{-2} \mathrm{~K}^{-1}
$$

This value is approximately five times lower than $2 B$. Then, instead of (27), we assume an expression for $\lambda_{R}$ as

$$
\lambda_{R}=\frac{2 B}{\sigma_{A}} \frac{f(r) R_{t}}{c_{1} \mathrm{e}^{-H / H} \text {. }}
$$

Using (35) with $(15 \mathrm{a}-\mathrm{c})$ the vertical integration of the rate of the generalized entropy 
production, equation (12), is

$$
\sigma=\frac{Q \cdot Q}{\lambda_{Q} T^{2}}+\frac{R \cdot R}{\lambda_{R} T^{2}}+\nabla \cdot H-\nabla \cdot\left(\frac{R}{T}\right)
$$

where the dimensionless gradient operator takes the form

$$
\begin{aligned}
& \nabla=a_{x}\left(1-x^{2}\right)^{1 / 2} \frac{\partial}{\partial x} \\
& \nabla \cdot=a_{x} \frac{\partial\left(1-x^{2}\right)^{1 / 2}}{\partial x}
\end{aligned}
$$

and

$$
\lambda_{R}=\frac{2 B}{\sigma_{A} D(H)}
$$

with $D(H)$ as

$$
D(H)=\frac{e^{H / H_{p}}}{c_{1} H} \int_{R_{\mathrm{t}}}^{R_{S}} d r r^{2} f(r) .
$$

Thus, all the variables in (36) are only functions of $t$ and $x$, so the entropy production can be expressed as

$$
\sigma=\frac{\boldsymbol{Q} \cdot \boldsymbol{Q}}{\lambda_{Q} T^{2}}+\frac{\boldsymbol{R} \cdot \boldsymbol{R}}{\lambda_{R} T^{2}}+\nabla \cdot \boldsymbol{H}-\frac{\nabla \cdot \boldsymbol{R}}{T},
$$

where the second term of the rhs is the radiative additional term in reference to the classical expression of the entropy production. The radiative flux of entropy is related with the radiative energy flux through effective temperatures, $T_{e},[13,27,39-41]$. Thus, we have

$$
\nabla \cdot H=\frac{4}{3} \frac{\nabla \cdot R}{T_{e}}
$$

where $T_{e}$ for the long-wave radiation corresponds to the atmospheric temperature, equation (31), and for the short-wave corresponds to the Sun photosphere temperature $(\sim 5777 \mathrm{~K})$.

The value of $\sigma_{A}$ is calculated considering a global radiative transfer equation:

$$
-\sigma_{A} \boldsymbol{R}=\frac{1}{c} \frac{d \boldsymbol{R}}{d t}
$$

As in a global system the first law of thermodynamics does not include the contribuJ. Non-Equilib. Thermodyn. $1998 \cdot$ Vol. $23 \cdot$ No. 1 
tion of the heat flux. Hence the absorption coefficient related to ' $\sigma_{A}$ is

$$
\sigma_{A}=-\frac{B}{\mathrm{c} \boldsymbol{R} \cdot \boldsymbol{a}_{r}} \frac{d T}{d t}+\frac{1}{\mathrm{cR} \cdot \boldsymbol{a}_{\mathrm{r}}} \frac{d S a}{d t}
$$

However, with the aim of simplifying the additional term in (40), as we have done in (35), we do not take into account the short-wave radiation in (43). The expression of $\lambda_{R}$ used is

$$
\lambda_{R}=-\frac{2 c(A+B T)}{D(H) \frac{d T}{d t}}
$$

where in the cases treated in Section $4, \lambda_{R}$ has been taken as the absolute value of equation (44). This assumption is made in order to simplify the importance of the additional term for the entropy production. We should have in mind that the 1-dimensional model only gives us qualitative results.

On the other hand, the coefficient $D(H)$ is evaluated by comparison of the divergence of the radiation field along the vertical with observations. Thus, assuming the function $f(r)$ as

$$
f(r)=\frac{r-R_{t}}{r^{2}} c_{1} e^{-\left(r-R_{t}\right) / H} p
$$

the value of $\nabla \cdot R(t, x)$ to the level $r$ from equation (15c) conveniently weighted is

$$
\left.\nabla \cdot \boldsymbol{R}(t, x)\right|_{r}=\boldsymbol{R}(t, x) \frac{r-R_{t}}{H R_{t}} c_{1} e^{-\left(r-R_{t}\right) / H_{p}}
$$

Equation (46) can be related to the total adiabatic heating in the atmosphere. The maximum values of this parameter depend on the latitude with a range approximately of $11 \mathrm{Wm}^{-2}$ at the equator to $6 \mathrm{~km}$ height and $-18 \mathrm{Wm}^{-2}$ at the pole to. $3 \mathrm{~km}$ height above the surface [42].

From (46) we obtain $H_{p}$ as the height where the radiative divergence has its maximum value. Then, defining $c_{2}=H / H_{p}, c_{1}$ is approximately

$$
c_{1} \approx 0.4 c_{2} R_{t} e
$$

where the factor 0.4 is accepted in view of the values of $\boldsymbol{R}(t, x)$ attained.

On the other hand, we show in Figure 1 the variation of $\nabla \cdot R(t, x)$ obtained in (46) as a function of height. The two axis are normalized with respect to their corresponding maximum values. We can see that an increase in $c_{2}$ implies the maximum in the radiative divergence to get closer to the surface. Assuming the atmospheric height about $60 \mathrm{~km}$, including the troposphere and the stratosphere, and taking only into account the long-wave radiation, because we have neglected the short-wave contribution in the calculation of $\lambda_{R}$, the value of the constant $c_{2}$ would be about 20 and, consequently from (39), $D(H)$ would take a value of $10^{+10} \mathrm{~m}$. Following the data record 


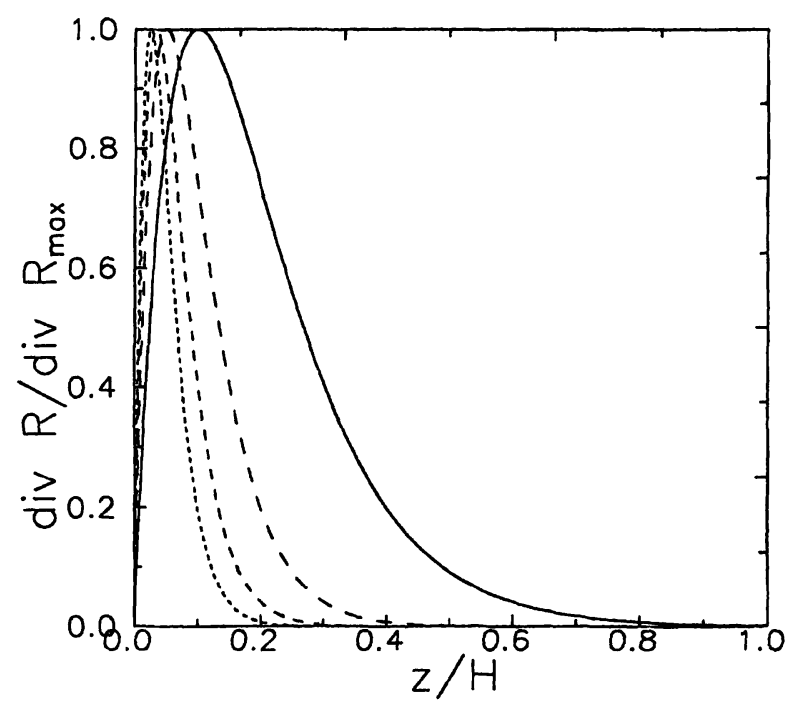

Fig. 1: Radiative divergence versus atmospheric height normalized by their maximum values $(H=60 \mathrm{~km})$. Due to the assumptions done in the calculus, $\lambda_{R}$ is mainly related with the long-wave contribution. According with the profiles of adiabatic heating observed in the atmoshpere [42], a value of $c_{2} \approx 20$ can be accepted $\left(-c_{2}=10 ; \cdots \cdots c_{2}=20 ; \cdots \ldots \ldots . \cdot \cdot\right.$ $\left.c_{2}=30 ; \cdots \cdots \cdots c_{2}=40\right)$.

by Peixoto and Oort [42], it corresponds to a maximum of about $3 \mathrm{~km}$ above from the surface. Higher values of $c_{2}$ could be admitted assuming a higher width for the atmosphere (e.g., $c_{2}=30$ for $H=90 \mathrm{~km}$ ) and vice-versa.

Although the radiative additional term in equation (40) vanishes when the system arrives at the steady state, its contribution can play a determinant role in the behaviour of the time evolution of entropy production. Thus, in the next sections we will see that high values of $c_{2}$ can modify completely the classical results. Nevertheless, according to the observations, we accept as a realistic value for $c_{2}$ that one indicated above.

\section{Results}

In this section we present the results of the time evolution of the classical and the generalized entropy production. In both cases, equation (40) has been applied. In reference to the extended contribution, the term $\lambda_{R}$ can be calculated through a meridional integration taking into account the latitudinal dependence (44). However, it can be approximated by a 'global' value of $\lambda_{R}$ obtained from (44), considering the mean value of temperature, so then we have

$$
\lambda_{R}=-\frac{2 c\left(A+B T_{0}\right)}{D(H) \frac{d T_{0}}{d t}} .
$$

Equation (48) and the global integration of (44) are clearly different expressions since (48) has no latitudinal dependence. The results of the entropy production with both values of $\lambda_{R}$ will be compared and analyzed in two distinct cases. 


\section{A. Ice-free earth}

In this case, the equations for the evolution of $T_{0}$ and $T_{2}$ are uncoupled. Therefore, the most simple model of an earth without ice-caps is to fix suitably the value of the global temperature. This fact allows two types of evolution for the rate of entropy production depending on the initial value assumed for $T_{2}$. Thus, for an initial value of $T_{2}$ greater than that corresponding to stationary conditions there will be a monotonical increase of entropy production. The introduction of the radiative fluxes as independent variables can change this behaviour because the additional term in (40) has always a positive contribution. Nevertheless, in the particular case where the global temperature is assumed to be constant, it is not possible to choose the value of $\lambda_{R}$ as (48). Thus, we have chosen for $\lambda_{R}$ the expression obtained in (44). Numerical results are depicted in Figure 2. The first case shows the evolution of a generilized expression of entropy production with a value of $\lambda_{R 0}=10^{-8} \mathrm{~s}^{-1}$, with $\lambda_{R 0}$ defined as

$$
\lambda_{R 0}=\frac{2 c}{D(H)} .
$$

Then, we have considered a value of $D(H) \sim 10^{+16} \mathrm{~m}$ corresponding to a value of $c_{2} \approx 35$. If we accept the value of $\lambda_{R 0}$ obtained by comparison with the observations, calculated at the end of the preceding section, $\lambda_{R 0}$ would be about $10^{-2} \mathrm{~s}^{-1}$. With this high value of $\lambda_{R 0}$ the radiative additional term can be neglected and the difference in the behaviour of the generalized and the classical expressions would be governed by the response time used in the heat flux term. However, aiming to observe the modifications produced by the additional radiative term, we will assume lower values of $\lambda_{R 0}$ even if these values are not expected for the climate system in the present state.

The second case depicted in Figure 2, corresponds to a lower value on $\lambda_{R 0}\left(10^{-9} \mathrm{~s}^{-1}\right)$. We can observe the change in the behaviour of the rate of entropy production outside the steady state in reference to the classical expression emphasizing the weight of the additional term related to the introduction of the EIT theory in the study of the system.

\section{B. Earth with ice-caps}

In this section, $T_{0}$ is permanent linked to $T_{2}$ through the value of the ice-sheet edge equation (30). Applying, for simplicity, equation (48), the behaviour of the classical entropy production grows monotonically, arriving its maximum value at the steady state if the initial conditions are not far from the steady conditions. However, taking into account the generalized expression, the monotonical behaviour is clearly unsatisfied due to equation (48), where $\lambda_{R}$ depends on $T_{0}$. Thus, in all the extremal points of $T_{0}$ along the evolution, the generalized and the classical entropy production have the same value. Nevertheless, in the other points, when $T_{0}$ evolves, the additional term of the generalized expression will provoke a higher value of the rate of entropy production with respect to its classical value. Assuming $\lambda_{R}$ as in (48), consecutive events of increasing and decreasing of entropy production with a minimum value equal to the classical one will be obtained depending on the non-monotonical evolution of $T_{0}$. 


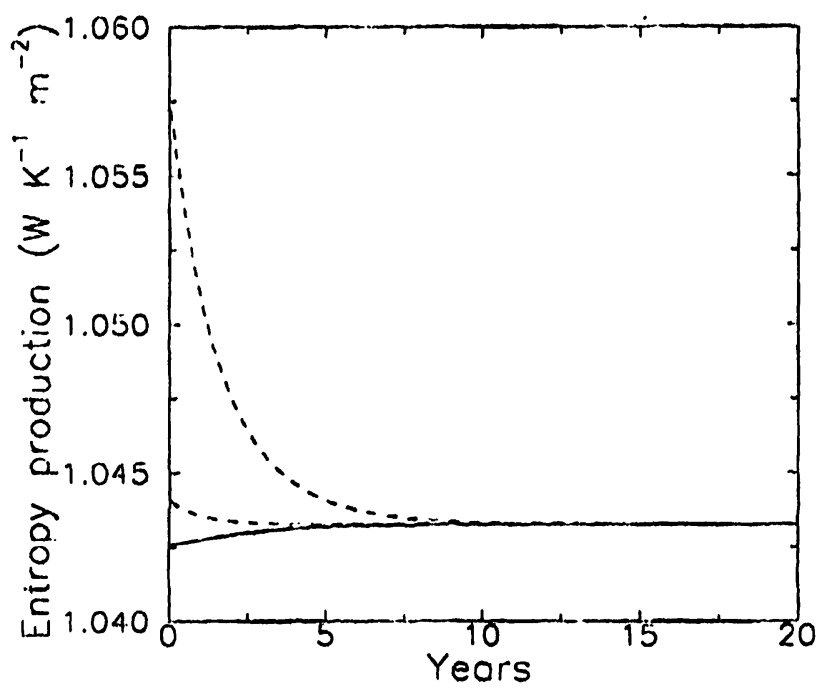

Fig. 2: Time evolution of the classical entropy productions $(-)$ compared with two different gencralized entropy productions with $\lambda_{p 0}=10^{-8} \mathrm{~s}^{-1}(\ldots \ldots \ldots)$ and $\lambda_{p 0}=10^{-9} \mathrm{~s}^{-1}$ $(\cdots \cdots \cdots \cdots, \ldots)$. The simple assumption of an ice-free earth is considered. Increasing the value of $\lambda_{R \mid D}$ there is a modification of the monotonical growing observed in the classical entropy production, with the possibility to change the steep of the time evolution curve. Initial conditions: $T_{0}=14.46^{\circ} \mathrm{C}$ invariable along the time evolution, $T_{2}=-16^{\circ} \mathrm{C}$. The initial heat flux is obtained applying Fourier's law at initial conditions. Other values: $\tau_{Q}=30$ days and the radiative parameters as indicate in Section 3.

Using (44), we show in Figure 3 the time-evolution of the classical and generalized expressions for three different values of $\lambda_{R}: \lambda_{R O}=10^{-8} \mathrm{~s}^{-1}, \lambda_{R O}=10^{-9} \mathrm{~s}^{-1}$ and $\lambda_{R V}=10^{-10} \mathrm{~s}^{-1}$. The initial conditions are shown in the figure corresponding to an initial global temperature equal to the present but with a higher difference of temperatures between the pole and the equator, considering, also, an expected response time of 30 days for the heat flux. We can observe the change of the monotonical behaviour obtained with the classical expression using low values of $\lambda_{R o}$, lower than $10^{-7} \mathrm{~s}^{-1}$. For the present state the additional radiative term does not imply quantitative modifications of the evolutions of the entropy production. For the three cases considered, there is a broken point originated by the same reasons given in reference to the application of (48) for the expression of $\lambda_{R}$. This fact is related to the importance of the additional term of the entropy production and to the assumption of the dissipative fluxes as independent variables outside equilibrium. Moreover, we have to point out that the assumption of a Maxwell-Cattaneo type equation for the heat flux can also modify the structure of the classical solutions. Thus, Pujol and Llebot [38] have shown that an increase of the response time used would provoke self-sustained oscillations, even without consideration of the additional radiative term, without the possibility to arrive at any steady state. It is due to the form of (19) and the coupling of the two compounds of the temperature implied by the value of the ice-sheet edge, equation (30). In the model used, then, the heat and radiative fluxes have a different influence on the time evolution. While the radiation part can modify the classical expression by changing the steepness of the time evolution, the heat flux can imply self-sustained oscillations for high values of the response time. 


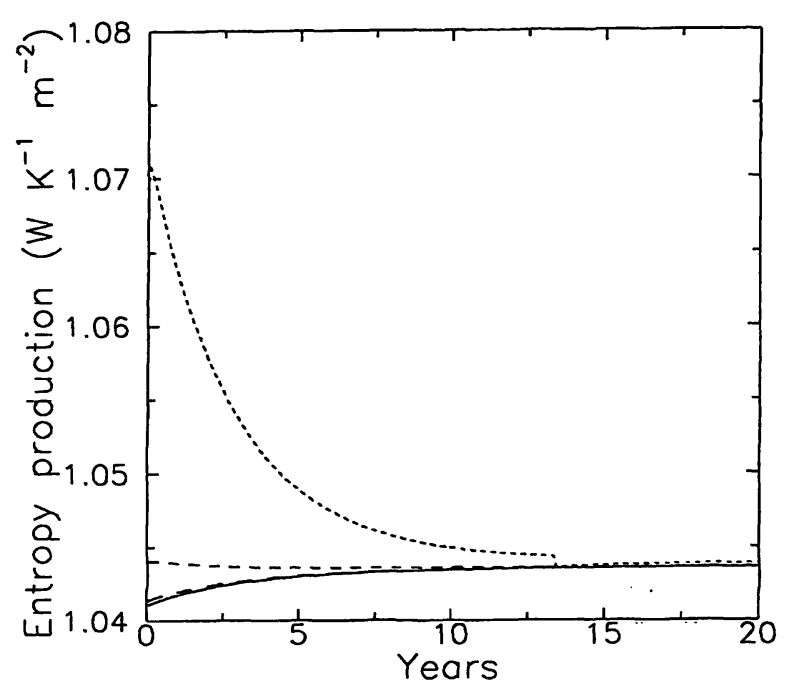

Fig. 3: Time evolution of the classical entropy production $(-)$ compared with three different cases for the generalized entropy production with $\lambda_{R 0}=10^{-8} \mathrm{~s}^{-1}(-\cdots \cdot-\cdot \cdot), \lambda_{R 0}=10^{-9} \mathrm{~s}^{-1}$ $(\cdot \cdot \cdot+\cdot \cdot+\cdot \cdot \cdot \cdot)$ and $\lambda_{R 0}=10^{-10} \mathrm{~s}^{-1}(\cdots \cdots \cdots \cdots \cdots \cdots \cdot \cdots)$. It is considered an earth with ice-caps and it is assumed a latitudinal integration to obtain $\lambda_{R}$. Initial conditions: $T_{0}=14.9^{\circ} \mathrm{C}, T_{2}$ $=-30^{\circ} \mathrm{C}$. The initial heat flux is obtained applying Fourier's law, at initial conditions. Other values: $\tau_{Q}=30$ days and the radiative parameters as indicate in Section 3 .

\section{Second differential of the entropy}

Usually, the stability study of a thermodynamic system at stationary conditions is done by using the second differential of the entropy, $\delta^{2} s$, taken as a Lyapunov functional [32]. Since $\delta^{2} s$ has a well defined sign (negative), $d\left(\delta^{2} s\right) / d t$ must be positive if the conditions of stability at the steady state are required. In the climate system, Nicolis and Nicolis [23] pointed out the possible use of $\delta^{2} s$ in a simple one-dimensional model.

However, we notice how the consideration of the dissipative fluxes, heat and radiative, in $\delta^{2} s^{*}$ can modify completely the behaviour of this parameter in reference to the classical expression, and, then, vary the applicability of the stability criteria. This fact has been largely commented by Jou et al. [24] where several explicit applications of the convexity requirement for the extended entropy have been considered. The results show how the condition of $\delta^{2} s^{*}$ to be negative definite implies a critical value for the dissipative fluxes within the framework of EIT. Thus, the range where $\delta^{2} s^{*}$ is not definite negative is related with length scales shorter than the mean-free path and characteristic times smaller than the collision time. This fact shows the self-consistency of EIT as a thermodynamic theory with a wider scope than classical CIT.

From the generalized Gibbs equation (1) and with the values obtained in (7a-b), the generalized expression of the second differential of the extended entropy takes the form

$$
\begin{aligned}
\delta^{2} s^{*}= & -C\left(\frac{\delta T}{T}\right)^{2}-\frac{\tau_{Q}}{\lambda_{Q} T^{2}}(\delta Q)^{2}-\frac{\tau_{R}}{\lambda_{R} T^{2}}(\delta R)^{2}+\frac{4 \tau_{Q}}{\lambda_{Q} T^{3}} Q \cdot \delta Q \delta T \\
& +\frac{4 \tau_{R}}{\lambda_{R} T^{3}} R \cdot \delta R \delta T-\frac{3 \tau_{q}}{\lambda_{Q} T^{4}} Q^{2}(\delta T)^{2}-\frac{3 \tau_{R}}{\lambda_{R} T^{4}} R^{2}(\delta T)^{2}
\end{aligned}
$$


Here we have neglected the third order differentials. In absence of radiation $(\boldsymbol{R}=0)$ equation (50) is the second differential of the extended entropy, including the conductive heat flux, widely analyzed, by Jou et al. [24], Criado-Sancho and Llebot [35]. Then, from (50), the knowledge of the radiative additional term implies an assumption over the parameter $\tau_{R} / \lambda_{R}$, that can be evaluated from (23) and (38) as

$$
\frac{\tau_{R}}{\lambda_{R}}=\frac{D(H)}{2 c B} .
$$

We adopt for the present atmospheric state, the value of $D(H)$ used in Section 3. Thus, $\tau_{R} / \lambda_{R} \approx 100 \mathrm{~m}^{2} \mathrm{~K} \mathrm{~s}^{2} \mathrm{~J}^{-1}$ is considered and, therefore, the additional term in (50) due to the radiative contribution can be neglected $\left(\tau_{q} / \lambda_{q} \approx 4 \cdot 10^{6} \mathrm{~m}^{2} \mathrm{~K} \mathrm{~s}^{2} \mathrm{~J}^{-1}\right.$, $\mathrm{C}=3.138 \cdot 10^{8} \mathrm{~m}^{2} \mathrm{~K} \mathrm{~s}^{2} \mathrm{~J}^{-1}$ ). In this case, $\delta^{2} s^{*}$ in an ice-free earth evolves in a convex form whereas the classical expression is not well-behaved for high values of the response time. For an earth with ice-caps, the assumption of the generalized entropy also enlarges the applicability of $\delta^{2} s^{*}$ as a stability criteria in reference to the classical expression. However, for high values of the response time both expressions lose their convex behaviour [24]. The time evolution of $\delta^{2} s^{*}$ with higher values of $\tau_{R} / \lambda_{R}$ has been studied with the intention of setting a better understanding of the behaviour of the additional radiative terms.

As in the preceding section we will treat two different models:

\section{A. Ice-free earth}

We have assumed a value of the parameter $\tau_{R} / \lambda_{R}=10^{+6} \mathrm{~m}^{2} \mathrm{~K} \mathrm{~s}^{2} \mathrm{~J}^{-1}$ corresponding to $c_{2} \approx 30\left(\lambda_{R 0} \sim 10^{-6} \mathrm{~s}^{-1}\right)$ in order to understand the role of the radiation field in the treatment of the second differential of the generalized entropy as a criteria of stability at the steady state. With usual values of the response time, the classical expression and the generalized one without the radiative part are well-behaved. When including the additional radiative contribution to the generalized case, $\delta^{2} s^{*}$ is behaved nonmonotonically (i.e., non-convex evolution). However, for values of $\tau_{R} / \lambda_{R}$ lower than those considered (e.g., the present state), the behaviour of $\delta^{2} s^{*}$ is closely related to the case where only the heat flux contribution is considered and, using the generalized entropy in this case, the range of applicability of the macroscopic theory is enlarged.

\section{B. Earth with ice-caps}

The value assumed for $\tau_{R} / \lambda_{R}$ in this case implies an important change of the behaviour of $\delta^{2} s^{*}$. For a response time of 30 days and with $\tau_{R} / \lambda_{R}=10^{+6} \mathrm{~m}^{2} \mathrm{~K} \mathrm{~s}^{2} \mathrm{~J}^{-1}$ the classical and the generalized entropy without the additional radiative term evolve in a similar manner, namely growing monotonically, and hence implying the stability of the steady state. On the other hand, the evolution of the generalized entropy with the contribution of the radiative part, is not a convex function in the initial stages due to the high variation experimented for the ice-sheet edge. In Figure 4, the influence of an increase of the factor $\tau_{R} / \lambda_{R}=10^{+8} \mathrm{~m}^{2} \mathrm{~K} \mathrm{~s}^{2} \mathrm{~J}^{-1}$ can be seen. In this figure we plot the evolution of the classical expression for $\delta^{2} s(T)$, the generalized entropy assuming the heat flux with 


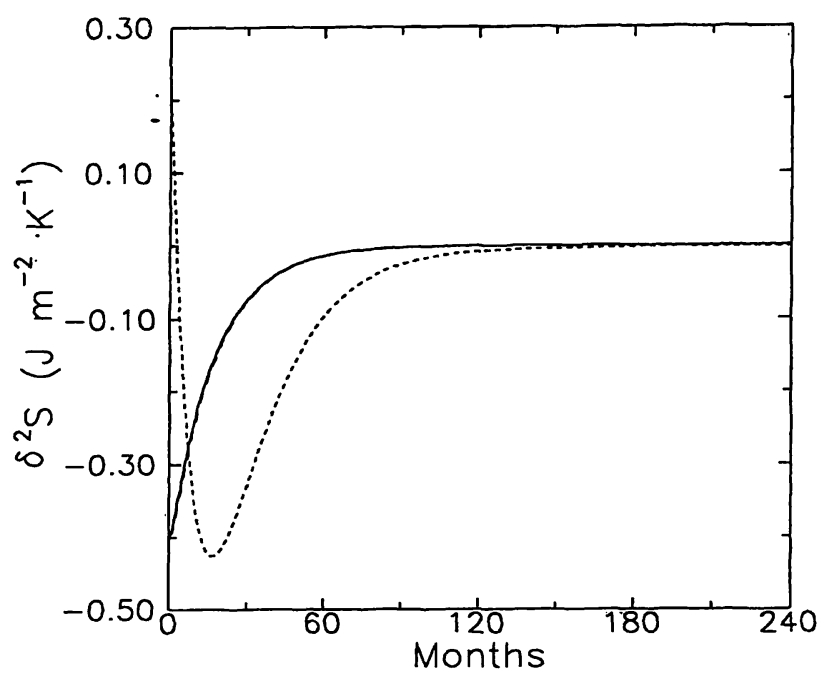

Fig. 4: Time evolution of the second differential of entropy assuming an earth with ice-caps. The classical (-) and the generalized expressions without the additional radiative part $(--.---)$ are very similar $\left(\tau_{Q}=30\right.$ days). The generalized expression assuming the radiative contribution $(\cdots \cdots \cdots \cdots \cdots \cdots \cdots)$ changes its sign for $\tau_{R} / \lambda_{R}=10^{+8} \mathrm{~m}^{2} \mathrm{Ks}^{2} \mathrm{~J}^{-1}$. It is not adequate to use $\delta^{2} s^{*}$ as a Lyapunov's functional. However, for low values of $\tau_{R} / \lambda_{R}$ (e.g., the present state) the results are similar to (1) enlarging the applicability of $\delta^{2} s^{*}$ in reference to the classical expression for high values of $\tau_{Q}$.

a delay time of 30 days $\left(\delta^{2} s^{*}(T, Q)\right)$, and the generalized entropy with radiative and heat flux contributions $\left(\delta^{2} s^{*}(T, \boldsymbol{Q}, \boldsymbol{R})\right)$. While for the two former expressions the behaviour is very similar, the generalized case taking into account the additional radiative term, does not have a single sign. Therefore, $\delta^{2} s^{*}(T, Q, R)$ can not be taken as a Lyapunov's functional and its expression is not suitable for determining the stability of the steady state due to its alternating sign. In comparison, the classical expression always is negative definite. However, as in the case of an ice-free earth, a lower value of $\tau_{R} / \lambda_{R}$ would imply a behaviour for $\delta^{2} s^{*}$ similar to that obtained with the introduction of the heat flux as independent variable. Hence, the generalized expression would increase the range of applicability in which $\delta^{2} s^{*}$ may be considered as a Lyapunov functional in reference to the classical expression.

An increase of the response time $\tau_{Q}$, does not modify the structure of the solutions commented. Thus, an indefinite sign of $\delta^{2} s^{*}$ is also obtained when the value of $\tau_{R} / \lambda_{R}$ corresponds to $10^{+8} \mathrm{~m}^{2} \mathrm{~K} \mathrm{~s}^{2} \mathrm{~J}^{-1}$, using a response time of 2.5 years. Such behaviour can be understood as high values of $\tau_{R} / \lambda_{R}$ require higher order terms in the development of the radiative part in the generalized Gibbs equation. This reasoning follows the same lines as in cases when the Maxwell-Cattaneo equation is not convenient due to using high values of the response time, and second and higher order terms must be taken into account.

\section{Extremal entropy production}

In Section 4 we have studied the behaviour of the time evolution of entropy production for the classical and the generalized cases. It is interesting to know whether or not the 
system satisfies an extremum principle of the entropy production, i.e., if along every state of the time evolution of the system, the values of the entropy production are extremal. In order to obtain a criteria for describing the possible extremal values of the rate of entropy production in general cases, we will develop, following the ideas of Nicolis and Nicolis [23], a variational principle at the steady state.

Using (40) at the steady state with equation (41) for the value of the radiation entropy flux, we obtain the global entropy production as

$$
\sigma=\frac{1}{3} \frac{A+B T-273 B}{T}-\frac{4}{3} \frac{S a}{T_{s}}+\frac{S a}{T}+Q \cdot \nabla \frac{1}{T} .
$$

From this equation we observe that $\sigma$ is only a function of $Q, \partial Q / \partial x$ and $x$ in stationary conditions since the temperature is only a function of $x$. Hence, we can calculate the heat flux $Q$ extremizing the global rate of entropy production using variational techniques [43]. The values for the heat flux resulting from extremizing the thermal part only and the difference between the radiative entropy flux and the radiative energy contribution of the entropy are also obtained. Thus, the expression of the extremal heat flux in terms of the radiative parameters are as follows

$$
\begin{aligned}
& \nabla \cdot Q=g(x)-\frac{\int_{0}^{1} d x g(x)}{\int_{0}^{1} d x(g(x))^{1 / 2}}(g(x))^{1 / 2} \\
& \nabla \cdot Q=g(x)-\int_{0}^{1} d x g(x) \\
& \nabla \cdot Q=g(x)-\frac{\int_{0}^{1} d x g(x)}{\int_{0}^{1} d x\left(g(x)-\frac{4(A-B 273)}{3}\right)^{1 / 2}}\left(g(x)-\frac{4(A-B 273)}{3}\right)^{1 / 2} .
\end{aligned}
$$

These three expressions correspond respectively to extremize the thermal part, the total expression for the entropy production and the difference between the radiative flux and the radiative energy contribution of the entropy production. In $(53 a-c)$ the parameter $g(x)$ takes the form

$$
g(x)=S a-A+273 B \text {. }
$$

The results of the heat flux deduced by means of extremal considerations, equations $(53 \mathrm{a}-\mathrm{c})$, are shown in Figure 5. The change in the bểhaviour at $72^{\circ}$ latitude is due to the beginning of the ice-cap and, in consequence, a constant value of the albedo.

Equation (53c) is equivalent to extremize

$$
\nabla \cdot\left(\boldsymbol{H}+\frac{\boldsymbol{R}}{T}\right)
$$

J. Non-Equilib. Thermodyn. $\cdot 1998 \cdot$ Vol. $23 \cdot$ No. 1 


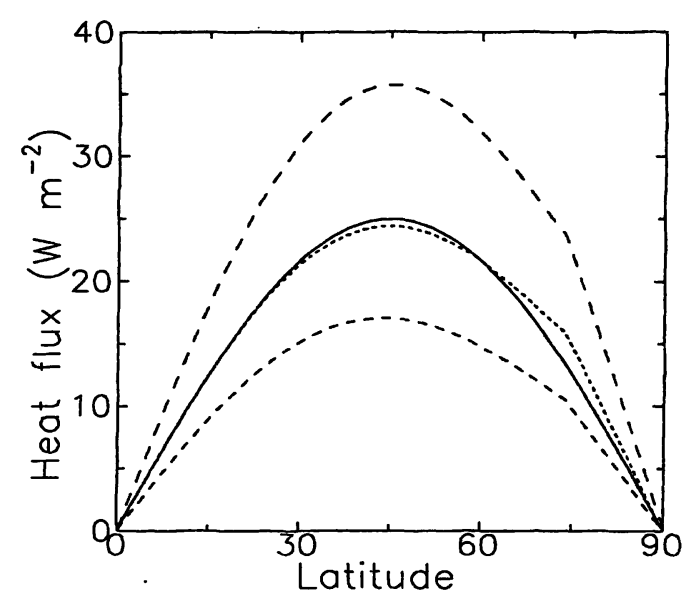

Fig. 5: Latitudinal dependence of heat flux at the steady state obtained through extremizing the

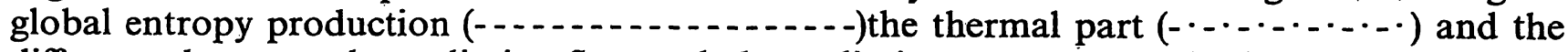
difference between the radiative flux and the radiative energy contribution of the entropy $(\cdots \cdots \cdots \cdots \cdots+$ The solid line indicates the heat flux used in the model. The change on the behaviour through $72^{\circ}$ is due to beginning of the ice-caps with a constant value of the albedo.

because in the system, the radiation and the gradient of temperature are assumed to be perpendiculars. If the radiation field would behave as the matter field, (55) would be twice the contribution of the global entropy production in the steady state and, therefore, the results of extremizing would not vary it in reference to equation (53b).

Nevertheless, we can approach the radiative flux of entropy in terms of the radiative flux of energy, equation (41), and then (55) can be written as

$$
\nabla \cdot\left\{\frac{7}{3} \frac{\boldsymbol{R}_{L}}{T}-\boldsymbol{R}_{s}\left(\frac{1}{T}+\frac{4}{3} \frac{1}{T_{s}}\right)\right\},
$$

where the subscripts $L$ and $S$ indicate the long-wave and the short-wave contribution respectively and $T_{s}$ is the Sun's photosphere temperature. Extremizing (56) implies an assumption of giving more weight to the long-wave than to the short-wave radiation. In fact, $a$ priori, there is no apparent reason for this assumption but we will show that the results obtained are in excellent agreement with the values for the heat flux assumed in the model $[3,13,23]$.

With the aim to obtain extremal values of the entropy production along the time evolution, one is tempted to apply expressions $(53 \mathrm{a}-\mathrm{c})$ outside the steady state. For the second case we show in Figure 6 the time evolution of the entropy production assuming the classical expression (very similar to the generalized entropy production with $\left.\lambda_{R O} \sim 10^{-2} \mathrm{~s}^{-1}\right)$, the time evolution of the radiative entropy fluxes $\nabla \cdot \boldsymbol{H}$, and its corresponding extremal value. The rate of entropy production increases monotonically till the steady state where its value is equal to that reached for the divergence of the radiative entropy fluxes. The extremal value along the evolution is close to the rate of entropy production and, then, it can be understood that the system is, even far from the 


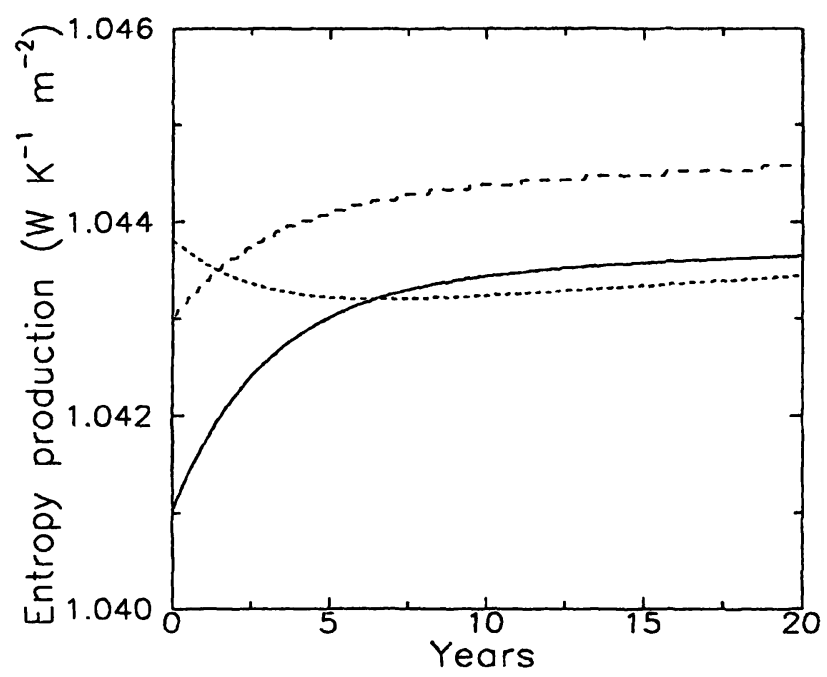

Fig. 6: Time evolution of the classical entropy production (-), very similar to the generalized entropy with a value of $\lambda_{R \theta} \sim 10^{-2} \mathrm{~s}^{-1}$ and $\tau_{Q}=30$ days, compared with the extremal expression (52b) obtained at the steady state (-....-.). It is also shown the time evolution of the radiative fluxes of the system $\nabla \cdot \boldsymbol{H}(\ldots \ldots \ldots)$ that in the stationary conditions would coincide with the entropy production. Initial conditions and other values as in Figure 3.

steady state, close to the conditions of an extremal on the global entropy production but not on the radiative entropy fluxes across the boundaries of the system.

On the other hand, due to the good approach of (55) to the present observation of the heat flux, we have calculated its time evolution directly from equation (53c). We have also applied the variational technique outside equilibrium assuming an expansion in Legendre polynomials for the temperature and considering the time variation of the temperature as independent of the heat flux. Equation (53c) then, takes the form

$\nabla \cdot Q=g(x)-C \frac{d T}{d t}-\frac{\int_{0}^{1} d x\left(g(x)-C \frac{d T}{d t}\right)}{\int_{0}^{1} d x\left(g(x)-\frac{4(A-B 273)}{3}\right)^{1 / 2}}\left(g(x)-\frac{4(A-B 273)}{3}\right)^{1 / 2}$.

Let us emphasize that with this assumption, the results concerning extremals of Figure 6 are not changed. In Figure 7 we also show the time evolution of the expression obtained by means of (53c). At the steady state, the three curves are very close, with a value higher than that corresponding to the entropy production due to a major global value of the long-wave radiation entropy than the short-wave contribution. Far from the steady state, the expression obtained from (53c) presents important variations to the curve calculated. However, using (57) the agreement along the evolution is quite good and better than that obtained in Figure 6 for the radiative contribution in reference to the global entropy production, showing that the system in any state seems to satisfy an extremal behaviour of (55), independently of the structure of its time evolution. 


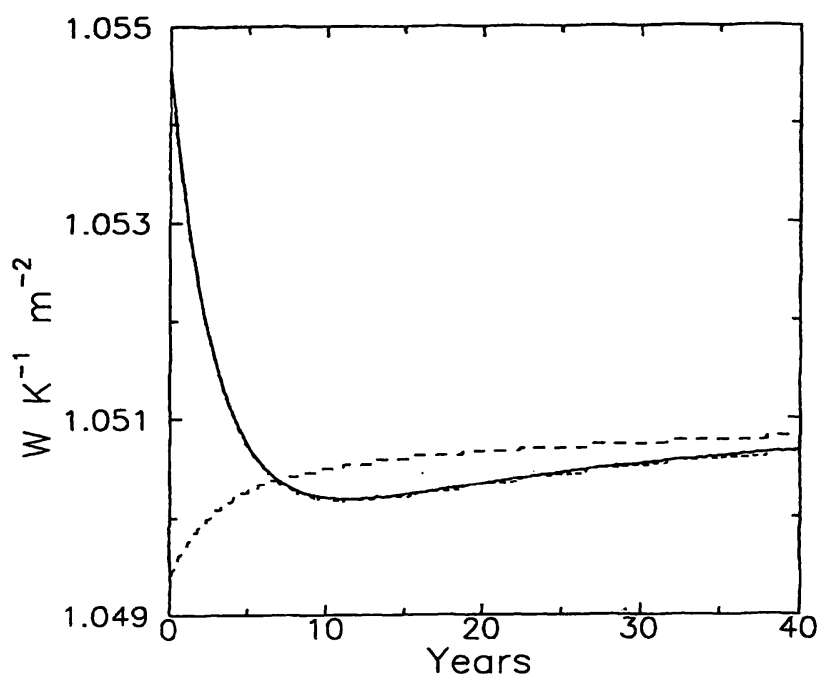

Fig. 7: Time evolution of $\nabla \cdot(H+R / T)(-)$ compared with the extremal curves obtained from equation (52c) (-.......-) and (56) $(. \ldots \ldots \cdots)$. It can be seen the good agreement for the last case with the values observed. The values for the constants are the same as in Figure 6.

\section{Summary}

The global aim of this paper has been to develop an expression for the entropy production in a non-equilibrium system with matter and radiative fluxes. We have used this fluxes as independent variables, developing a generalized Gibbs equation following the Extended Irreversible Thermodynamics (EIT) theory [24] that has been successfully applied in many systems. We have taken into account a Maxwell-Cattaneo type equation for the phenomenological laws of the heat and the radiative fluxes, that in the later case can be related to the radiative transfer equation [33, 36-37]. A generalized expression for the rate of entropy production outside equilibrium has been obtained with the addition of a new term related with the radiative part. At the steady state the generalized expression takes the form of the classical entropy production.

The equations obtained have been applied in a simple EBM climate model. Nevertheless, in order to obtain the additional radiative contribution we have developed a 2-dimensional model that integrated along the vertical reduces to the classical one. This procedure allows to know the radiation field necessary to develop the generalized theory. It is shown that the role of the radiative field in the entropy production can be decisive for its behaviour. We have seen that for certain values of $\lambda_{R}$, the generalized expression clearly differs from the classical results. Following the development done in the present paper, high variations of the values of $\lambda_{R}$ could be obtained choosing properly the atmospheric parameters, although by comparison with the vertical distribution obtained in the model and the values observed for the vertical adiabatic heating in the atmosphere, we have assumed a value of $\lambda_{R}$ for which the additional radiative term due to the EIT theory does not substantially modify the classical results. Nevertheless, our choice of the value of the additional radiative term not necessarily is the most precise due to the simplifications inherent in the model developed. The study 
carried out in the present paper can give us an idea about the possible contributions of the dissipative fluxes to the entropy production and the relationship of these variables with the states of the climate. Moreover, variations of the response time of the heat flux would also produce substantial changes of the behaviour of the rate of entropy production [38].

Following the parallelism of studying the climate as a simple thermodynamic system, we have applied the second differential of the entropy as a criteria for determining the stability of the steady state. In this case, the introduction of the radiation field can modify completely the results with regard to the classical and to those related to the generalized expression of the entropy production without radiative part. Thus, for low values of the radiative parameters $\left(\tau_{R} / \lambda_{R}=100 \mathrm{~m}^{2} \mathrm{~K} \mathrm{~s}^{2} \mathrm{~J}^{-1}\right.$ as the present state) the results for the generalized expression of the entropy production are the same as those corresponding to considering only the heat flux as an independent variable. Nevertheless, using high values of $\tau_{R} / \lambda_{R}, \delta^{2} s^{*}$ shows a non-well definite sign for an earth with ice-caps and then, it cannot be used as a Lypaunov functional. (Jou et al. [24] had pointed out the fact that the sign of the second differential of the generalized entropy is not always well definite.)

In the study of the climate many authors have considered some constraints in order to decrease the number of free variables in the system. Thus, Paltridge [15-16] in his box model assumed that the climate system is close to the maximum dissipation rate related with the matter entropy production. In the EBM variational methods and principles easily can be applied as Nicolis and Nicolis [23] did. Here, we have shown an extension of these ideas calculating the heat flux corresponding to extremizing the thermal part, the global entropy production and the difference between the radiative flux and the radiative energy contribution of the entropy production. The last expression shows a good agreement with the values observed for the present heat flux. Moreover, we have developed the last two expressions to obtain an extremal value far from the steady conditions, showing a good agreement with the time evolution values. The results indicate that the climate system follows an extremal principle of $\nabla \cdot(\boldsymbol{H}+\boldsymbol{R} / T)$ independent of the behaviour of its time evolution. If we assume this hypothesis we have a restriction about the system, the knowledge of the heat flux along the evolution not being necessary. This constraint permits to decide wether or not the diffusive approach is a reasonable application for the climate system. Thus from (57) and (20) we obtain the latitudinal dependence of the temperature through the variational principle as

$$
T(x)=T_{0} \frac{\left(g(x)-\frac{4(A-B 273)}{3}\right)^{1 / 2}}{\int_{0}^{1} d x\left(g(x)-\frac{4(A-B 273)}{3}\right)^{1 / 2}},
$$

where $T_{0}$ is the global temperature. Assuming an expansion in Legendre polynomials for the temperature obtained through the extremal conditions

$$
T(x)=T_{0}+T_{2 e} P_{2}(x),
$$


$T_{2 e}$ takes the form

$$
T_{2 e}(x)=T_{0}\left\{\frac{\left(g(x)-\frac{4(A-B 273)}{3}\right)^{1 / 2}}{\int_{0}^{1} d x\left(g(x)-\frac{4(A-B 273)}{3}\right)^{1 / 2}}-1\right\} \frac{1}{P_{2}(x)} .
$$

In the EBM models $T_{2}$ has not a latitudinal dependence, but here, we have taken into account the value that would correspond to it applying extremal considerations. Then, one can admit a value of $T_{2}$ as the averaged temperature obtained through a latitudinal integration of $T_{2 e}$ and obtain its time evolution once the variations of $T_{0}$ and $x_{s}$ are known. The results show that this evolution is very similar compared to that one observed from the classical diffusive EBM. Then, the application of Fourier's law, and in consequence, of a Maxwell-Cattaneo type equation, can be accepted as a good approximation in the dynamics of the system. This fact reaffirms the validity of the expression of the rate of entropy production outside equilibrium found here.

\section{Acknowledgement}

This work has been partially supported by the Spanish Ministry of Science and Education under contract CL195-1867.

\section{References}

[1] Budyko, M.I., The effect of solar radiation variations on the climate of the earth, Tellus, 21 (1969), 611-619.

[2] Sellers, W. D., A global climatic model based on the energy balance of the earthatmosphere system, J. Appl. Met., 8 (1969), 392-400.

[3] North, G.R., Theory of energy-balance models, J. Atmos. Sci., 32 (1975), 2033-2043.

[4] North, G. R., Analytical solution to a simple climate model with diffusive heat transfer, J. Atmos. Sci., 32 (1975), 1301-1307.

[5] Bhattacharya, K. M., Ghil, M., Vulis, I.L., Internal variability of an energy-balance model with delayed albedo effects, J. Atmos. Sci., 39 (1982), 1747-1773.

[6] Sellers, W. D., A new global climatic model, J. Appl. Met., 12 (1973), 241-253.

[7] Pollard, D., A coupled climate-ice sheet model applied to the Quaternary Ice Ages, J. Geophys. Res., 88 (1983), 7705-7718.

[8] Harvey, L. D., A semianalytic energy balance climate model with explicit sea ice and snow physics, J. Climate, 1 (1988), 1065-1085.

[9] Gallée, H., van Ypersele, J.P., Fichefet, Th., Tricot, Ch., Berger, A., Simulation of the last glacial cycle by a coupled, sectorially averaged climate-ice sheet model 1 . The climate model, J. Geophys. Res., 7 (1991), 13139-13161.

[10] Stocker, T. F., Wright, D. G. Mysak, L. A., A zonally averaged, coupled ocean-atmosphere model for paleoclimates studies, J. Climate, 5 (1992), 773-797.

[11] Cess, R. D., Potter, G. L., Blanchet, J. P., Boer, G. J., Ghan, S. J., Kiehl, J. T., Le Treut, H., Li, Z.-X., Liang, X.-Z., Mitchell, J.F.B., Morcrette, J.-J., Randall, D. A., Riches, M., Roechner, E., Schlesse, U., Slingo, A., Taylor, K. E., Washington, W. M., Wetherald, R. T., Yagai, I., Interpretation of cloud-climate feedback as produced by 14 atmospheric general circulation models, Science, 214 (1989), 513-516. 
[12] Delworth, T. S., Manabe, S., Stouffer, R. J., Interdecadal variations of the thermohaline circulation in a coupled ocean-atmosphere model, J. Climate, 6 (1993), 1993-2011.

[13] Li, J., Chýlek, P., Entropy in climate models. Part II: Horizontal structure of atmosphericentropy production, J. Atmos. Sci., 51 (1994), 1702-1708.

[14] Li, J., Chýlek, P., Lesins, G. B., Entropy in climate models. Part I: Vertical structure of atmospheric entropy production, J. Atmos. Sci., 51 (1994), 1691-1701.

[15] Paltridge, G. W., Global dynamicas and climate - a system of minimum entropy exchange, Quart. J. R. Met. Soc., 101 (1975), 475-484.

[16] Paltridge, G. W., The steady-state format of globl climate, Quart. J. R. Met. Soc., 104 (1978), 927-945.

[17] Paltridge, G. W., Climae and thermodynamic systems of maximum dissipation, Nature, 279 (1979), 630-631.

[18] Paltridge, G. W., Thermodynamic dissipation and the global climate system, Quart J. R. Met. Soc., 107 (1981), 531-547.

[19] Stephens, G. L., O’Brien, D. M., Entropy and climate. I: ERBE observations of the entropy production of the earth, Quart. J. R. Met. Soc., 119 (1993), 121-152.

[20] O'Brien, D. M., Stephens, G. L., Entropy and climate. II: Simple models, Quart. J. R. Met. Soc., 121 (1995), 1773-1796.

[21] Ziegler, H., An introduction to thermomechanics, North-Holland, Amsterdam-New YorkOxford, 1977.

[22] Grassl, $H$, The climate at maximum entropy production by meridional atmospheric and oceanic heat fluxes, Quart. J. R. Met. Soc., 107 (1981), 153-166.

[23] Nicolis, G., Nicolis, C., On the entropy balance of the earth-atmosphere system, Quart. J. R. Met. Soc., 106 (1980), 691-706.

[24] Jou, D., Casas-Vázquez, J., Lebon, G., Extended irreversible thermodynamics, SpringerVerlag, Berlin-Heidelberg-New York, 1996.

[25] De Groot, S. R., Mazur, P., Non-Equilibrium Thermodynamics, Dover, 1984.

[26] Essex, C., Radiation and irreversible thermodynamics of climate, J. Atmos. Sci., 41 (1984), 1985-1991.

[27] Essex, C., Minimum entropy production in steady stae and radiative transfer, Astrophys. J., 285 (1984), 279-293.

[28] Callies, U., Herbert, F., On the treatment of radiation in the entropy budget of the earth-atmosphere system, Dev. Atmos. Sci., 16 (1984), 311-329.

[29] Callies, U., Herbert, F., Radiative processes and non-equilibrium thermodynamics, J. Appl. Math. Phys., 39 (1988), 242-266.

[30] Mobbs S. D., Extermal principles for global climate models, Quart. J. R. Met. Soc., 108 (1982), 535-550.

[31] Shutts G. H., Maximum entropy production states in quasi-geostrophic dynamical models, Quart. J. R. Met. Soc., 107 (1981), 503-520.

[32] Prigogine, I., Etude thermodynamique des phénomènes irreversibles, Desoer, Liège, 1947.

[33] Fort, J., Llebot J. E., Radiative transfer in the framework of Extended Irreversible Thermodynamics, J. Phys. A, 29 (1996), 3427-3436.

[34] Ore, A., Entropy of radiation, Phys. Rev., 98 (1955), 887-888.

[35] Criado-Sancho, M., Llebot, J. E., Behaviour of entropy in hyperbolic heat conduction, Phys. Rev. E, 47 (1993), 4104-4107.

[36] Ciancio, V., Verhás, J., A thermodynamic theory for radiating heat transfer, J. Non-Equilib. Thermodyn., 15 (1990), 33-43.

[37] Ciancio, V., Verhás, J., A thermodynamic theory for heat radiation through the atmosphere, J. Non-Equilib. Thermodyn., 16 (1991), 57-65.

[38] Pujol, T., Llebot, J. E., Periodic solutions in low-dimensional climatic models, J. Climate, (1997) (submitted).

[39] Landsberg, P. T., Tonge, G., Thermodynamics of the conversion of diluted radiation, J. Phys. A, 12 (1979), 551-562.

[40] Lesins, G. B., On the relationship between radiative entropy and temperature distributions, J. Atmos. Sci., 47 (1990), 795-803. 
[41] Peixoto, J. P., Oort, A. H., de Almedia, M., Tomé, A., Entropy budget of the atmosphere, J. Geophys. Res., 96 (1991), 10981-10988.

[42] Peixoto, J. P., Oort, A. H., Physics of climate, American Institute : of Physics, New York, 1992.

[43] Goldstein, H., Classical mechanics, Addison-Wesley, Massachusetts. 1950.

Paper received: $1997-7-21$

Paper accepted: $1997-12-18$

T. Pujol

Grup de Física, Dept. de Ciències Ambientals

Facultat de Ciències

Universitat de Girona

Campus Montilivi

E-17071 Girona

Spain
Prof. Dr. J. E. Llebot

Dept. de Física

Facultat de Ciències

Universitat Autònoma de Barcelona E-08193 Bellaterra

Spain 